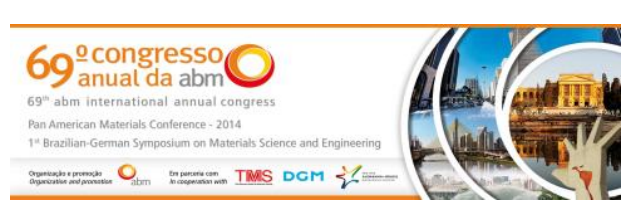

Tema: Mecânica de fratura e integridade estrutural

\title{
PROPAGAÇÃO DE TRINCA POR FLUÊNCIA NO AÇO ASTM A 297 GR HP MODIFICADO COM NIÓBIO*
}

\author{
Maria Cristina Adami Gatti ${ }^{1}$ \\ Cassius Olívio Figueiredo Terra Ruchert² \\ Levi de Oliveira Bueno ${ }^{3}$ \\ Waldek Wladimir Bose Filho ${ }^{4}$ \\ Dirceu Spinelli ${ }^{5}$
}

\section{Resumo}

Este trabalho tem por objetivo aplicar o conceito teórico e experimental fornecido pela mecânica da fratura dependente do tempo, para avaliar por meio do parâmetro de fratura $C^{*}$ suscetibilidade do aço ASTM A297 Gr HP-Nb, para resistir à propagação de trincas por fluência. Este aço é utilizado na fabricação de colunas de suporte de tubos radiantes de Unidades de Coqueamento Retardado, que operam por longos períodos de tempo em temperaturas elevadas. Os ensaios de fluência convencional e de propagação de trinca por fluência foram realizados na temperatura de $927^{\circ} \mathrm{C}$, de acordo com as recomendações das normas ASTM E139-11 e ASTM E1457-13. Dos resultados das curvas de fluência obtida para diferentes valores de tensões aplicadas foram determinadas as constantes, $A$ e $n$, da relação de Norton. Com os resultados obtidos da taxa de propagação de trinca por fluência, da/dt, em função do parâmetro $C^{*}$, foi possível ser modelada a equação $d a / d t=1,1 \times 10^{-3}\left(C^{*}\right)^{0,78}$. A morfologia da fratura observada na região de crescimento de trinca por fluência no estado estacionário apresentou modo de propagação interdentrítica, típica de aços com estrutura austenítica bruta de fusão.

Palavras-chave: Fluência; Propagação de trinca por fluência; Aço ASTM A297-Nb; Integral- $C^{*}$.

\section{CREEP CRACK PROPAGATION OF NIOBIUM MODIFIED ASTM A-297 GR HP STEEL Abstract}

This work aims to apply the theoretical and experimental concepts provided by timedependent fracture mechanics to assess, by means of fracture parameter $C^{*}$, the ability of steel ASTM A297 Gr HP modified with Niobium, to resist creep crack propagation. This material is used in the manufacture of steel support columns of radiant tubes of Retarded Coking Units to operate for long periods of time at high temperatures. The tests of conventional creep and creep crack propagation were held in the temperature of $927^{\circ} \mathrm{C}$, in accordance to ASTM E139-11 and E1457-13. From the results of creep curves obtained for different values of applied stress were determined the constants, $A$ and $n$, from Norton relationship. With the results obtained from the creep crack propagation rate $d a / d t$ as a function of the parameter $C^{*}$, the equation $d a / d t=1,1 \times 10^{-3}\left(C^{*}\right)^{0.78}$ could be modeled. Fracture morphology presented interdendritic propagation features in the steady-state creep region, typical of steels presenting as-cast austenitic structure.

Keywords: Creep; Creep crack propagation; ASTM A297 Gr HP-Nb steel; Integral-C*.

1 Engenheira Civil, Doutora, Pós-Doutoranda, Departamento de Engenharia de Materiais, Escola de Engenharia de São Carlos, Universidade de São Paulo, São Carlos, SP, Brasil.

2 Engenheiro Mecânico, Doutor, Professor Doutor, Departamento de Engenharia de Materiais, Escola de Engenharia de São Carlos, Universidade de São Paulo, São Carlos, SP, Brasil.

3 Engenheiro Mecânico, Doutor, Professor Adjunto, Departamento de Engenharia de Materiais da Universidade Federal de São Carlos, São Carlos, SP, Brasil.

4 Engenheiro Mecânico, Doutor, Professor Titular, Departamento de Engenharia de Materiais, Escola de Engenharia de São Carlos, Universidade de São Paulo, São Carlos, SP, Brasil.

5 Engenheiro Mecânico, Doutor, Professor Sênior, Departamento de Engenharia de Materiais, Escola de Engenharia de São Carlos, Universidade de São Paulo, São Carlos, SP, Brasil.

\footnotetext{
* Contribuição técnica ao 69 Congresso Anual da ABM - Internacional e ao 14ํㅡㄹ ENEMET - Encontro Nacional de Estudantes de Engenharia Metalúrgica, de Materiais e de Minas, 21 a 25 de julho de 2014, São Paulo, SP, Brasil.
} 


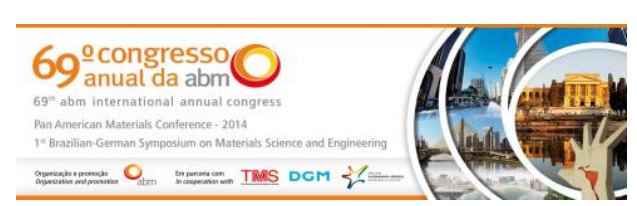

\section{INTRODUÇÃO}

A característica mais comum entre os aços inoxidáveis é o elevado teor de cromo, que provoca o aparecimento do fenômeno conhecido como passivação a estas ligas e sendo por isto, usados em ambientes altamente corrosivos e em temperaturas elevadas ou ambos. Tal como outras ligas, os aços inoxidáveis classificam-se como fundidos e trabalhados, sendo que estes últimos sofrem conformação mecânica para o condicionamento estrutural, antes da obtenção da forma final.

Os aços inoxidáveis fundidos da série $\mathrm{H}$, quando aplicados em temperaturas acima de $650^{\circ} \mathrm{C}$, devido ao alto teor de carbono em sua composição química apresentam uma evolução microestrutural conforme a temperatura de serviço aumenta e, desta forma, mantém a sua elevada resistência mecânica, particularmente sob o ponto de vista da resistência a fluência [1].

Entre os aços inoxidáveis fundidos resistentes a altas temperaturas destacam-se os graus HK e HP, que são extensivamente usados em aplicações estruturais em temperaturas de até $1150^{\circ} \mathrm{C}$, mantendo boa resistência à corrosão por gases quentes.

A composição básica de um aço HP exibe uma microestrutura típica de um aço como fundido resistente ao calor, consistindo de uma matriz austenítica circundada por carbonetos de cromo primários $\left(\mathrm{Cr}_{7} \mathrm{C}_{3}\right)$, principalmente na orientação interdendrítica, Figura 1.

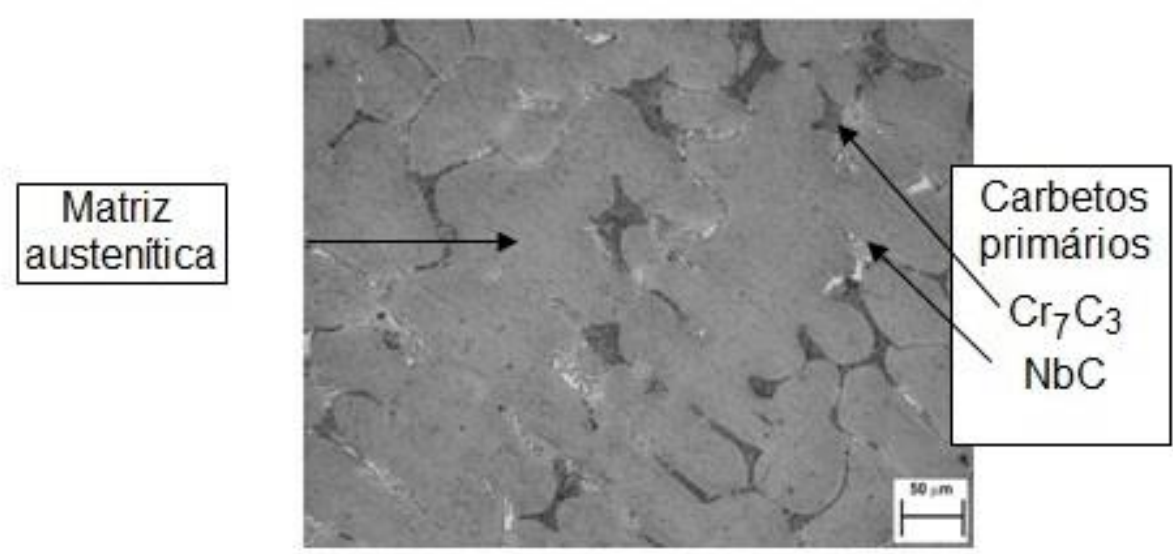

Figura 1. Fotomicrografia do aço atacado com reagente Água Régia [2].

Neste trabalho o comportamento de crescimento de trinca por fluência do aço inoxidável ASTM A297 Gr HP modificado com Nb foi investigado, usando o parâmetro de fratura $C^{*}$ da mecânica da fratura dependente do tempo. Para determinar as constantes do material necessárias para calcular o parâmetro de fratura $C^{*}$, ensaios de fluência convencionais e propagação de trinca por fluência foram realizados em vários níveis de tensões na temperatura de $927^{\circ} \mathrm{C}$. Com a utilização dos resultados experimentais obtidos, foi possível modelar uma relação entre a taxa de propagação de trinca por fluência da/dt em função do parâmetro de fratura $C^{*}$, para o aço inoxidável HP-Nb na temperatura de $927^{\circ} \mathrm{C}$.

\footnotetext{
* Contribuição técnica ao $69^{\circ}$ Congresso Anual da ABM - Internacional e ao 14ํㅡㄹ ENEMET - Encontro Nacional de Estudantes de Engenharia Metalúrgica, de Materiais e de Minas, 21 a 25 de julho de 2014, São Paulo, SP, Brasil.
} 


\section{MATERIAL E MÉTODOS}

\subsection{Material}

O material investigado foi o aço fundido ASTM A-297 Gr HP modificado com Nióbio na condição de como-fundido [3], cuja composição química está apresentada na Tabela 1 e as suas propriedades mecânicas do ensaio de tração na temperatura de $927^{\circ} \mathrm{C}$ estão mostradas na Tabela 2 [2].

Tabela 1. Composição química do aço ASTM A-297 Gr HP (\% em peso).

\begin{tabular}{cccccccccc}
\hline $\mathbf{C}$ & $\mathbf{S i}$ & $\mathbf{M n}$ & $\mathbf{P}$ & $\mathbf{S}$ & $\mathbf{C r}$ & $\mathbf{N i}$ & $\mathbf{M o}$ & $\mathbf{C u}$ & $\mathbf{N b}$ \\
\hline 0,40 & 1,10 & 1,11 & 0,018 & 0,004 & 25,7 & 35,8 & 0,085 & 0,088 & 1,01 \\
\hline
\end{tabular}

Tabela 2. Propriedades de tração do aço ASTM A-297 Gr HP ensaiado a 9270 C.

\begin{tabular}{|c|c|c|c|c|}
\hline $\begin{array}{c}\mathrm{E} \\
(\mathrm{GPa})\end{array}$ & $\begin{array}{c}\mathbf{S}_{\mathbf{r}} \\
(\mathrm{MPa})\end{array}$ & $\begin{array}{l}\mathrm{Se}_{\mathrm{e}(0,2 \%)} \\
(\mathrm{MPa})\end{array}$ & $\begin{array}{l}\text { AT } \\
(\%)\end{array}$ & $\begin{array}{l}\text { RA } \\
\text { (\%) }\end{array}$ \\
\hline 70,8 & 75,1 & 56,8 & 15,4 & 50,5 \\
\hline
\end{tabular}

\subsection{Métodos}

\subsubsection{Ensaio de fluência convencional}

Os ensaios de fluência foram realizados com tensões constantes de 36,40 , 50 , e $60 \mathrm{MPa}$ na temperatura de $927^{\circ} \mathrm{C}$, utilizando-se extensometria de alta temperatura (LVDT), para medida de deformação em função do tempo, de acordo com a norma ASTM E-139-11 [4]. A obtenção da curva de fluência para diferentes níveis de tensão possibilitou a determinação dos valores das taxas de fluência no estágio secundário ou estacionário, para a obtenção dos parâmetros $A$ e $n$ da relação de Norton dada pela Equação 1.

$$
\varepsilon_{s}=\mathrm{A} \sigma^{n}
$$

Onde $A$ é a constante característica do material, $n$ é o expoente de tensão e $\dot{\varepsilon}_{s}$ a taxa de fluência no estágio secundário.

A Figura 2 apresenta a geometria e dimensões do corpo de prova que foi utilizado nos ensaios de fluência convencional.

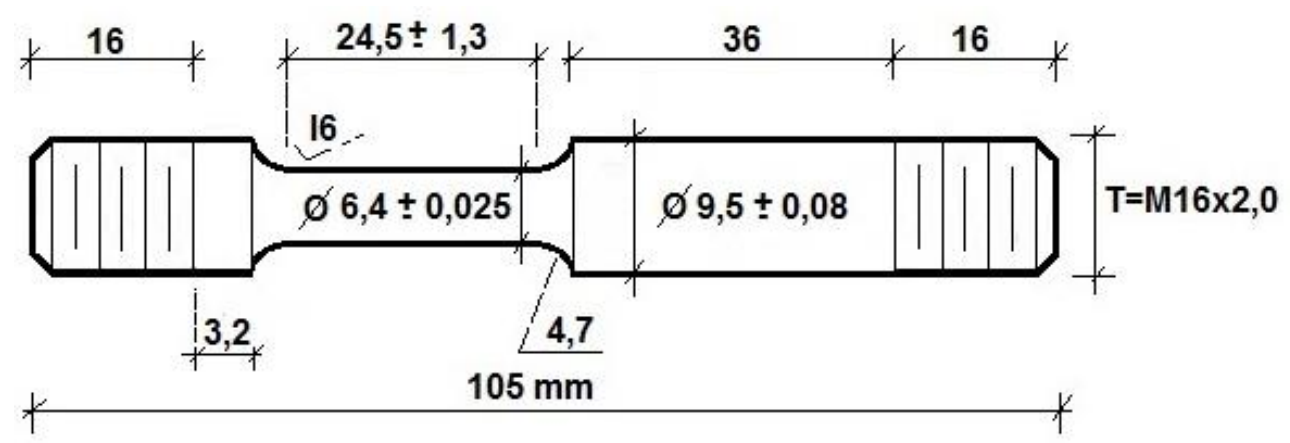

Figura 2. Geometria e dimensões do corpo de prova.

\footnotetext{
* Contribuição técnica ao $69^{\circ}$ Congresso Anual da ABM - Internacional e ao 14ํㅡㄹ ENEMET - Encontro Nacional de Estudantes de Engenharia Metalúrgica, de Materiais e de Minas, 21 a 25 de julho de 2014, São Paulo, SP, Brasil.
} 


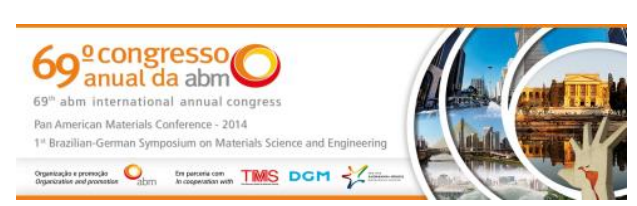

\subsubsection{Ensaios de propagação de trinca por fluência}

Os ensaios de propagação de trinca por fluência foram realizados na temperatura de $927^{\circ} \mathrm{C}$, em uma Máquina de Ensaio de Fluência que foi doado pela Empresa Engemasa - Engenharia e Materiais Ltda e adaptada pelo Núcleo de Ensaios de Material e Análise de Falha do Departamento de Engenharia de Materiais para operar segundo a norma ASTM E1457-13 [5]. Os corpos de prova usados nos ensaios foram do tipo $\mathrm{C}(\mathrm{T})$, com a geometria e dimensões conforme mostrada na Figura 3. Os ensaios foram realizados em dois corpos de prova $\mathrm{C}(\mathrm{T})$ pré-trincados por fadiga, que foram solicitados em dois níveis de carregamentos em cargas constantes, que produziram fatores de intensidades de tensões iniciais de 5,5 MPa $\mathrm{m}^{1 / 2}$ e 6,8 MPa m${ }^{1 / 2}$.

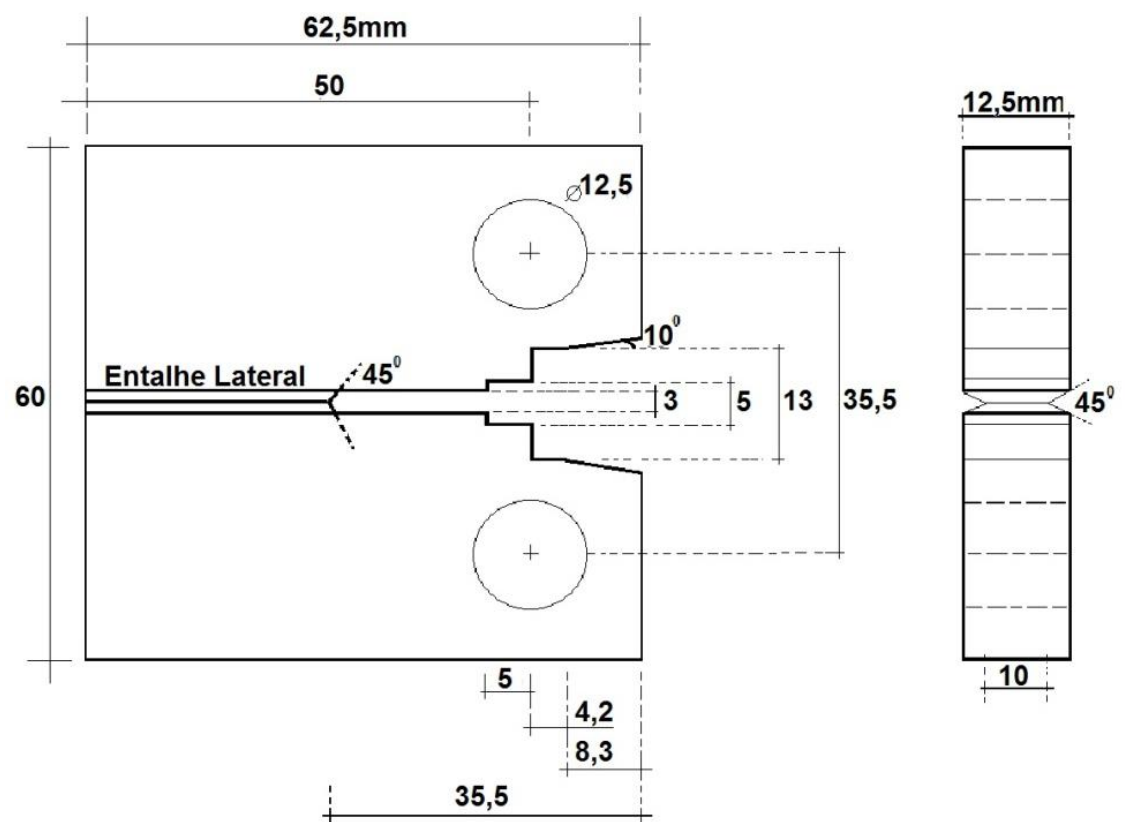

Figura 3. Geometria e dimensões do corpo de prova do tipo C(T).

O valor do parâmetro $C^{*}$ foi determinado por meio da Equação 2 mostrada a seguir.

$$
C^{*}=\frac{P \dot{V}^{D L C}}{B_{n}(W-a)} H^{D L C} \eta^{D L C}
$$

Onde $P$ é a carga aplicada, $W$ é a largura do corpo de prova, a é o comprimento de trinca, $B_{n}$ é a espessura líquida, $\dot{V} D L C$ é a taxa de deslocamento na linha de carga $(D L C)$ no estágio secundário de propagação, $H^{D L C}$ e $\eta^{D L C}$ são funções da geométrica do corpo de prova $\mathrm{C}(\mathrm{T})$ adotado no ensaio. A função $H^{D L C}$ foi calculada pela Equação 3 e a função $\eta^{D L C}$ foi obtida pelo valores da relação a/W, como especificados na Norma ASTM E1457-13 [5].

$$
H^{D L C}=\frac{n}{n+1}
$$

Onde $n$ é o expoente de tensão da relação de Norton obtido no ensaio de fluência convencional [4].

As medidas do crescimento da trinca por fluência foram realizadas por meio do método de diferença de potencial elétrico com descrito na norma ASTM E1457-13 [5]. Na Figura 4 estão localizados os pontos de entrada de corrente e saída de

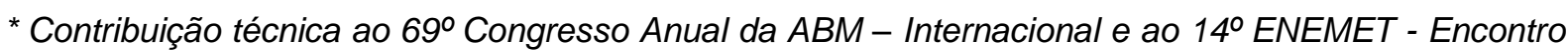
Nacional de Estudantes de Engenharia Metalúrgica, de Materiais e de Minas, 21 a 25 de julho de 2014, São Paulo, SP, Brasil. 


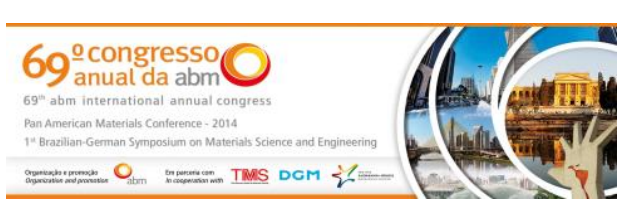

mínima $\varepsilon_{s}$ que foram determinadas para o mínimo valor da taxa de fluência no estágio secundário de propagação.

Na Figura 5 está mostrado o gráfico da variação da taxa mínima de fluência com a tensão aplicada, para determinação das constantes $n$ e $A$ da relação de Norton do material na temperatura de $927^{\circ} \mathrm{C}$.

Tabela 3. Resultados dos parâmetros de fluência na temperatura de $927^{\circ} \mathrm{C}$.

\begin{tabular}{ccccccc}
\hline $\begin{array}{c}\boldsymbol{\sigma} \\
(\mathrm{MPa})\end{array}$ & $\begin{array}{c}\dot{\boldsymbol{\varepsilon}}_{\boldsymbol{s}} \\
\left(\% \mathrm{~h}^{-1}\right)\end{array}$ & $\begin{array}{c}\mathbf{t}_{\mathbf{r}} \\
(\mathrm{h})\end{array}$ & $\begin{array}{c}\text { AR } \\
(\%)\end{array}$ & $\begin{array}{c}\text { RA } \\
(\%)\end{array}$ & $\log \left(\dot{\varepsilon}_{\boldsymbol{s}}\right)$ & $\log (\boldsymbol{\sigma})$ \\
\hline 36,0 & 0,001125 & 465,45 & 12,8 & 28,4 & $-1,949$ & 1,551 \\
\hline 40,0 & 0,048829 & 166,59 & 20,2 & 43,8 & $-1,311$ & 1,602 \\
\hline 50,0 & 0,266510 & 41,57 & 16,4 & 46,6 & $-0,574$ & 1,699 \\
\hline 60,0 & 2,319600 & 6,30 & 32,9 & 53,1 & $-0,365$ & 1,778 \\
\hline
\end{tabular}

Onde $\sigma$ : tensão aplicada; " $\varepsilon_{s}$ : taxa mínima no estado secundário; $t_{r}$ : tempo de ruptura; $A R$ : alongamento na ruptura; $R A$ : redução de área.

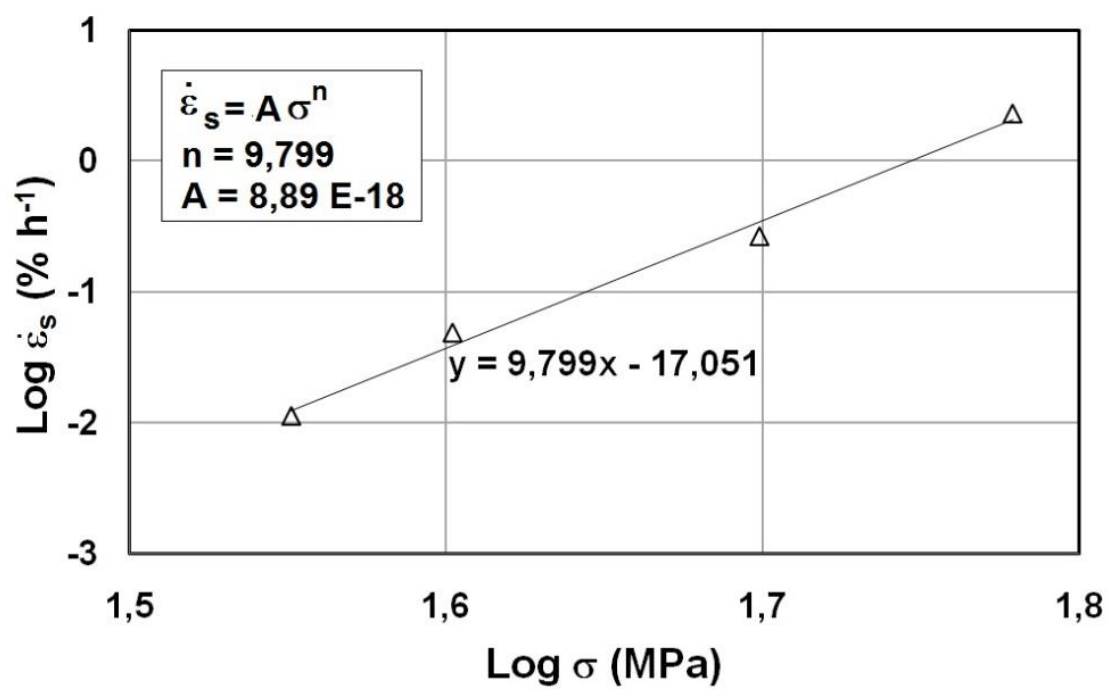

Figura 5. Variação da taxa mínima de fluência com a tensão.

Nota-se na Figura 5, que a relação de Norton ficou muito bem definida por uma linha reta, no diagrama log-log. Assim o expoente de tensão $n$ da relação de Norton obtido foi de 9,8 e a constante característica do material $A$ de $8,89 \mathrm{E}-18 \mathrm{MPa}^{-\mathrm{n}} \mathrm{h}^{-1}$.

\subsection{Ensaios de Propagação de Trinca por Fluência}

A Figura 6 apresenta o resultado obtido para o aumento do comprimento da trinca em função do tempo em dois fatores de intensidades de tensões iniciais $K_{i}$ de 6,8 $\mathrm{MPam}^{1 / 2}$ e 5,5 $\mathrm{MPam}^{1 / 2}$.

$\mathrm{Na}$ Figura 6 pode-se observar que o corpo de prova submetido ao fator de intensidade de tensão inicial $\left(K_{i}\right)$ de valor menor apresentou decréscimo da taxa de crescimento da trinca menor no estágio de propagação estável, quando comparada ao corpo de prova que foi solicitado por um fator de intensidade de tensão inicial $K_{i}$ maior.

\footnotetext{
* Contribuição técnica ao $69^{\circ}$ Congresso Anual da ABM - Internacional e ao 14ํㅡㄹ ENEMET - Encontro Nacional de Estudantes de Engenharia Metalúrgica, de Materiais e de Minas, 21 a 25 de julho de 2014, São Paulo, SP, Brasil.
} 

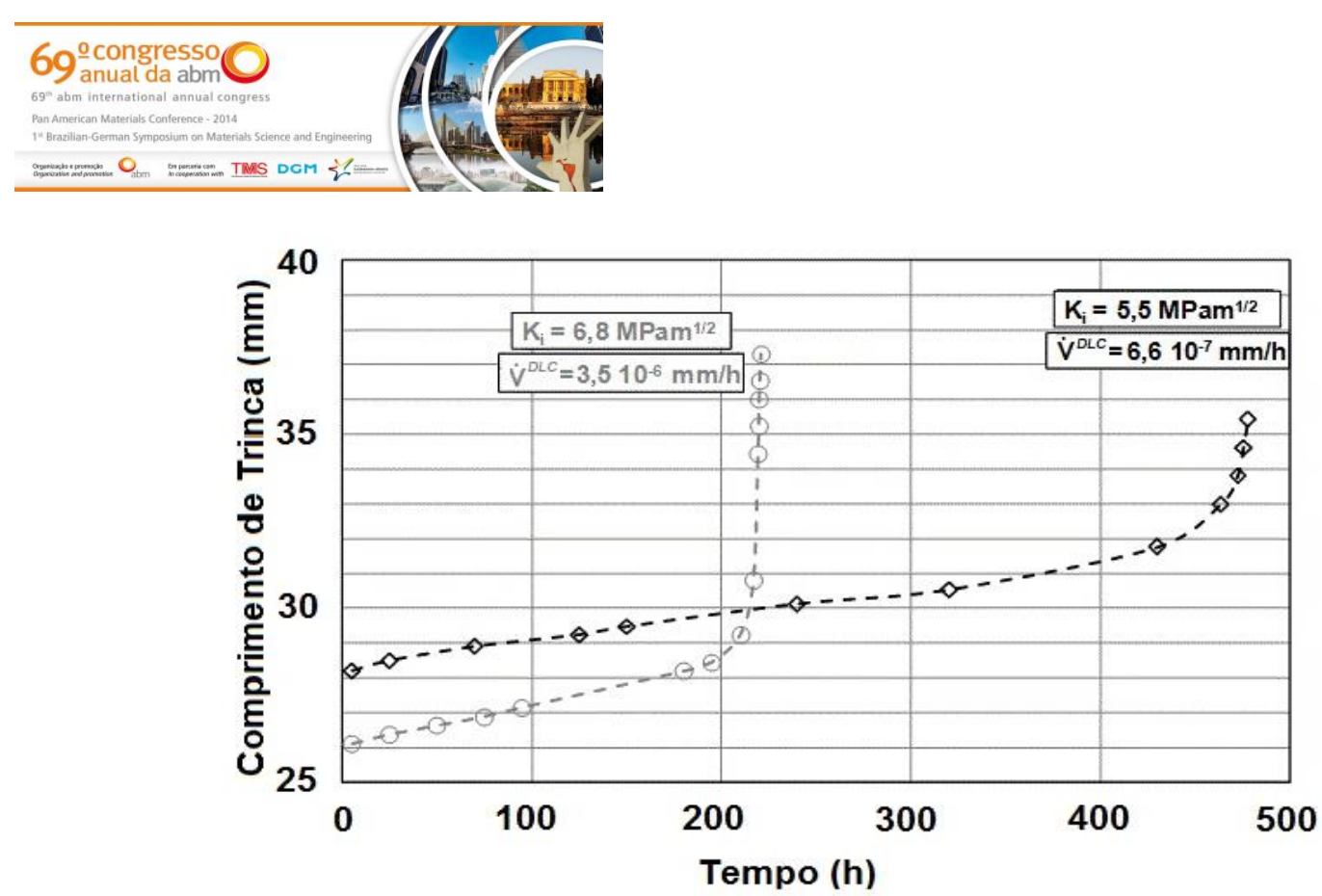

Figura 6. Curvas do aumento do comprimento de trinca em função do tempo do ensaio.

A Figura 7 apresenta as curvas que mostram o aumento do deslocamento da linha de carga em função do tempo para os dois fatores de intensidade de tensão iniciais aplicados. Por meio destas curvas foi possível determinar a relação entre a taxa de crescimento de trinca $d a / d t$ em função do parâmetro de fratura $C^{*}$ de intensidade de tensão dependente do tempo.

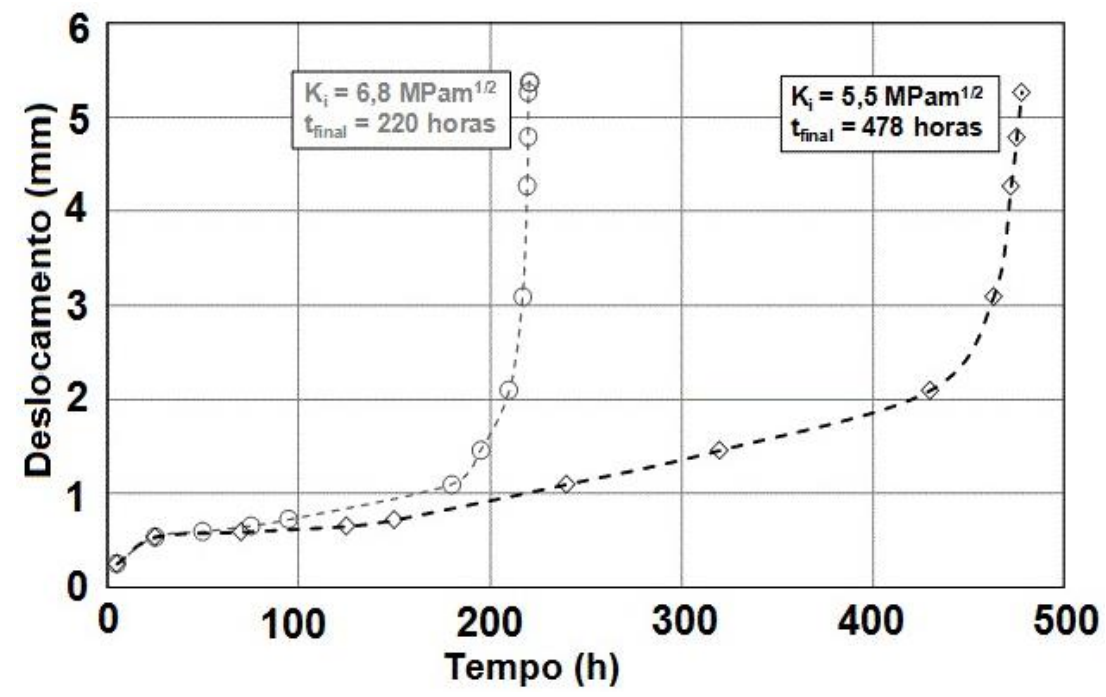

Figura 7. Curvas do aumento do deslocamento da linha de carga em função do tempo do ensaio.

A Figura 8 apresenta os resultados da taxa de propagação de trinca por fluência $d a / d t$ em função do parâmetro de fratura $C^{*}$, obtidos de acordo com o procedimento descrito no item 2.2.2. A relação $d a / d t$ versus $C^{*}$ pode ser representada pela Equação 5 descrita a seguir.

$$
d a / d t=1,1 x 10^{-3}\left(C^{*}\right)^{0,78}
$$

\footnotetext{
* Contribuição técnica ao 69ำ Congresso Anual da ABM - Internacional e ao 14ํㅡㄹ ENEMET - Encontro Nacional de Estudantes de Engenharia Metalúrgica, de Materiais e de Minas, 21 a 25 de julho de 2014, São Paulo, SP, Brasil.
} 

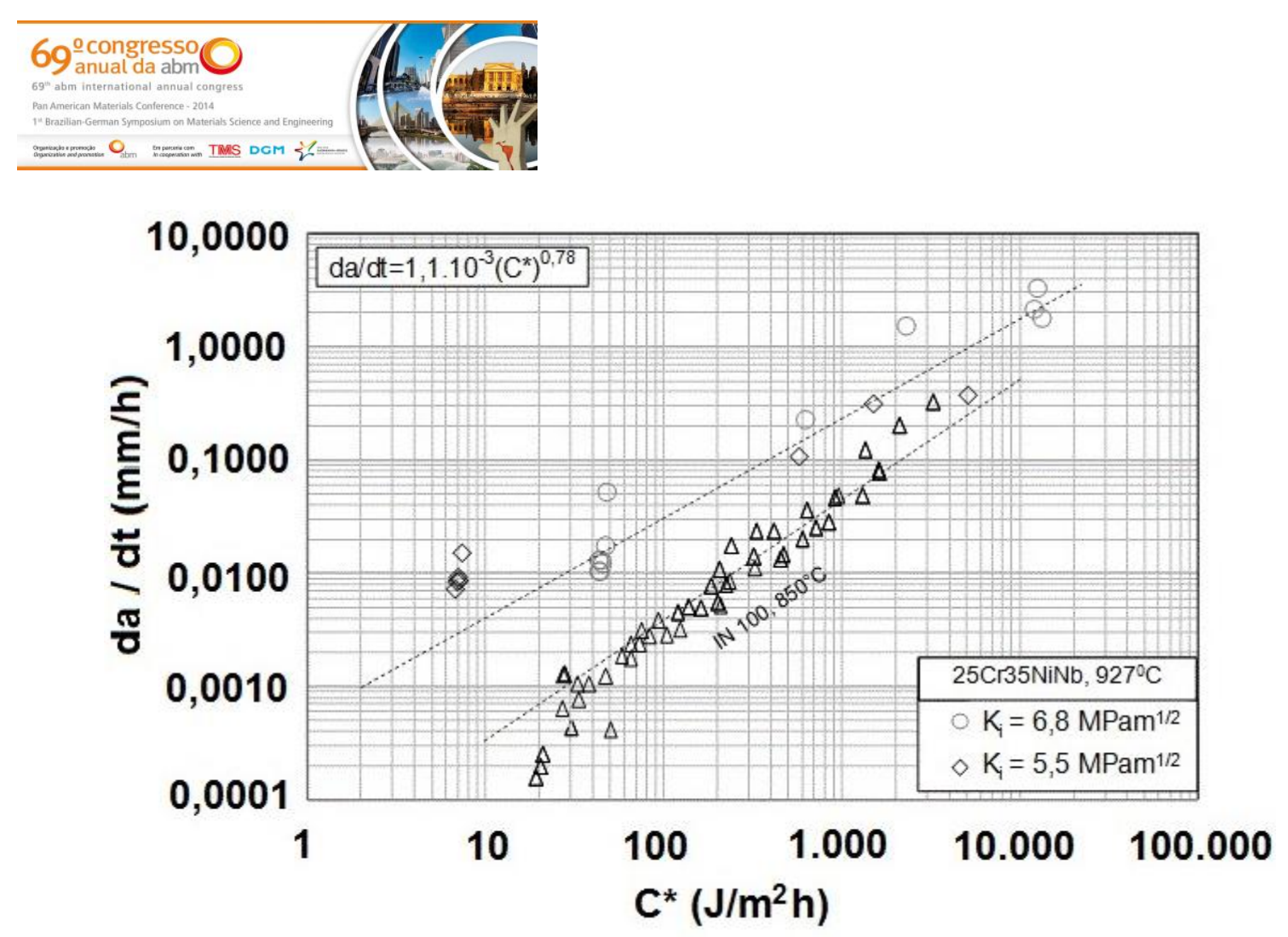

Figura 8. Relação entre a taxa de crescimento de trinca $d a / d t$ em função do parâmetro $C^{*}$.

Na Figura 8, também estão apresentados os resultados das taxas de propagação de trinca por fluência em função do parâmetro de fratura $C^{*}$, obtidos para uma superliga de Ni denominada de IN100 [6], para comparação. Da análise dos resultados obtidos neste trabalho, pode-se concluir que a liga ASTM A297 Gr HP-Nb, na condição bruta de fusão, apresentou taxas de propagação de trinca por fluência para valores baixos de $C^{*}$, de até 10 vezes maiores quando comparada a superliga IN100 na condição bruta de fusão.

As Figuras 9 e 10 apresentam os aspectos macro e micro fractográficos, obtidos pela técnica de MEV, das superfícies de fratura dos dois corpos de prova fraturados nos ensaios de crescimento de trinca por fluência, ensaiados a $927^{\circ} \mathrm{C}$.

Nas Figuras 9 e 10 a seta 1 indica a região da pré-trinca por fadiga onde pode-se notar um modo planar típico de propagação de trinca por fadiga. Já na região indicada pela seta 2 observa-se uma superfície de fratura com o aspecto típico de propagação lenta por fluência, onde se identifica um modo de propagação interdentrítico, bem como o processo severo de oxidação, devido ao longo tempo de permanência na temperatura de $927^{\circ} \mathrm{C}$.
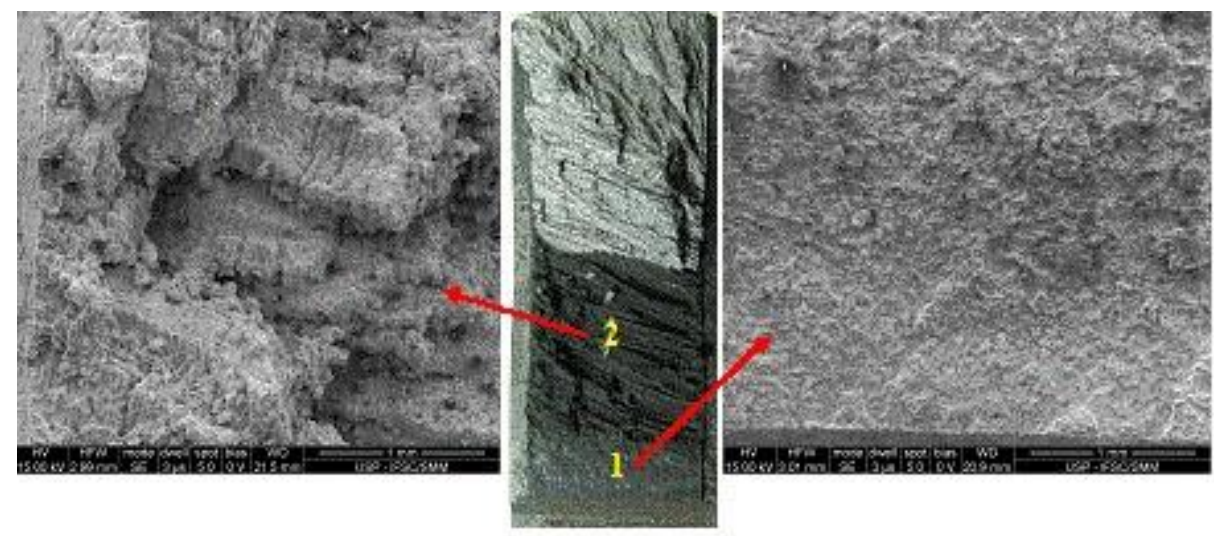

Figura 9. Aspectos macro e micro fractográficos obtidos em diferentes regiões na fratura do corpo de prova ensaiado com $K_{i}=6,8 \mathrm{MPam}^{1 / 2}$.

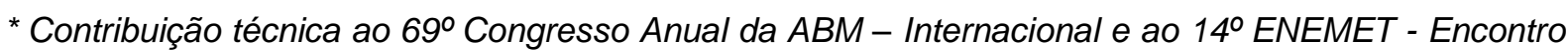
Nacional de Estudantes de Engenharia Metalúrgica, de Materiais e de Minas, 21 a 25 de julho de 2014, São Paulo, SP, Brasil.
} 

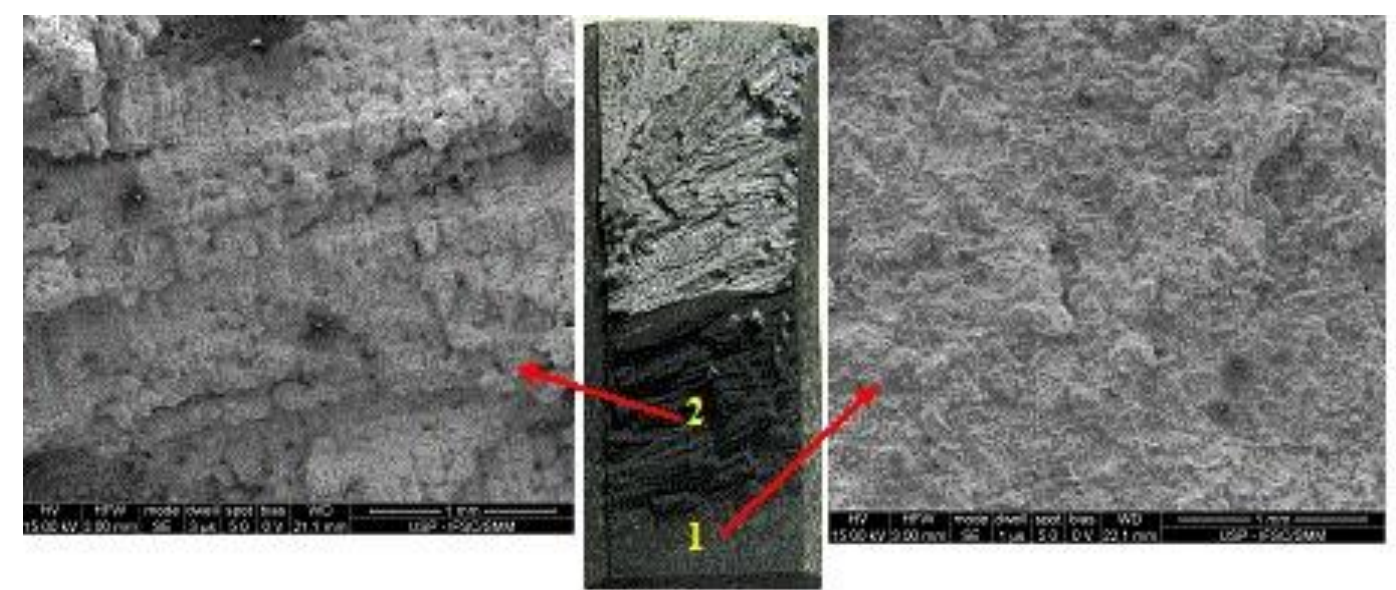

Figura 10. Aspectos macro e micro fractográficos obtidos em diferentes regiões na fratura do corpo de prova ensaiado com $K_{l}=5,5 \mathrm{MPam}^{1 / 2}$.

\section{CONCLUSÕES}

- $\quad$ A relação de Norton $\varepsilon_{s}=A \cdot \sigma^{n}$ ficou muito bem definida por uma linha reta, no diagrama $\log \left(\varepsilon_{s}\right)-\log (\sigma)$ e o expoente de tensão $n$ obtido foi de 9,8 e a constante característica do material $A$ obtida foi de $8,89 \mathrm{E}-18 \mathrm{MPa}^{-n} \mathrm{~h}^{-1}$.

- Nos ensaios de propagação de trinca por fluência observou-se que com a aplicação de fator de intensidade de tensão inicial $\left(K_{i}\right)$ menor proporcionou um decréscimo da taxa de crescimento da trinca no estágio estacionário, quando comparado o valor de $K_{i}$ maior. Este comportamento é o esperado para um material com estrutura no estado bruto de fusão.

- Dos resultados da taxa de propagação de trinca por fluência, $d a / d t$ em função do parâmetro de fratura $C^{*}$, obtidos pela técnica experimental desenvolvida neste trabalho foi possível modelar a equação $d a / d t=1,1 \times 10^{-3}\left(C^{*}\right)^{0,78}$, que poderá ser aplicada na previsão de vida de componentes fabricados com 0 material estudado.

- Deste trabalho pode-se também concluir que o aço ASTM A297 Gr HP-Nb, com microestrutura bruta de fusão, apresenta taxas de propagação de trinca por fluência de aproximadamente de 10 vezes maiores para baixos valores de $C^{*}$, quando comparada a superliga de níquel IN100, também na condição bruta de fusão.

- Na região de propagação da trinca por fluência, nota-se um modo de fratura com o aspecto típico de propagação interdentrítica, bem como um processo severo de oxidação, devido ao longo tempo de permanência na temperatura de $927^{\circ} \mathrm{C}$.

\section{Agradecimentos}

Os autores expressam os seus agradecimentos a Empresa Engemasa - Engenharia e Materiais Ltda pela doação da Máquina de Fluência e pelo fornecimento do aço ASTM A297 Gr HP-Nb e as agências de fomento à pesquisa CAPES (Proc. 23038038434/2008-33), CNPq (Proc. 301967/2010-5) e FAPESP pelo apoio financeiro.

\footnotetext{
* Contribuição técnica ao $69^{\circ}$ Congresso Anual da ABM - Internacional e ao 14ํㅡㄹ ENEMET - Encontro Nacional de Estudantes de Engenharia Metalúrgica, de Materiais e de Minas, 21 a 25 de julho de 2014, São Paulo, SP, Brasil.
} 


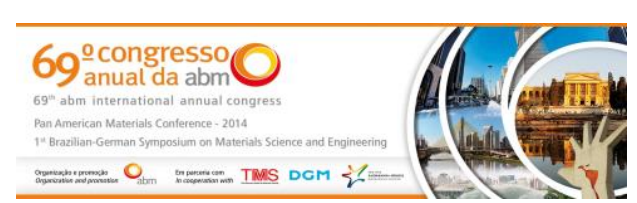

\section{REFERÊNCIAS}

1 Nunes FC, Almeida LH, Dille J, Delplancke JL, Le May I. Microstructural changes caused by yttrium addition to NbTi-modified centrifugally cast HP-type stainless steel. Materials Characterization. 2007;58(2):132-142.

2 Castro DBV, Gatti MCA, Ruckert COFT, Spinelli D, Bose Filho WW. Propriedades de tração e impacto do aço ASTM A-297 Gr HP modificado com nióbio, em altas temperaturas. In: Associação Brasileira de Metalurgia, Materiais e Mineração. Anais do 65ํㅡㄹ Congresso Internacional ABM; 2010; Rio de Janeiro, Brasil. São Paulo: ABM; 2010. p.512-523.

3 American Society for Testing and Materials. ASTM A-297-10: Standard Specification for Steel Castings, Iron-Chromium and Iron-Chromium-Nickel, Heat Resistant, for General Application. West Conshohocken; 2010.

4 American Society for Testing and Materials. ASTM E-139-11: Standard test methods for conducting creep, creep-rupture and stress-rupture tests of metallic materials. West Conshohocken; 2011.

5 American Society for Testing and Materials. ASTM E-1457-13: Standard test methods for measurement of creep crack growth times in metals. West Conshohocken; 2013.

6 Tabuchi M, Kobo K, Yagi K, Yokobori Jr., Fuji A. Results of a Japanese round robin on creep crack growth evaluation methods for Ni-base superalloys. Engineering Fracture Mechanics. 1999;62(1):47-60.

\footnotetext{
* Contribuição técnica ao $69^{\circ}$ Congresso Anual da ABM - Internacional e ao 14ํㅡㄹ ENEMET - Encontro Nacional de Estudantes de Engenharia Metalúrgica, de Materiais e de Minas, 21 a 25 de julho de 2014, São Paulo, SP, Brasil.
} 